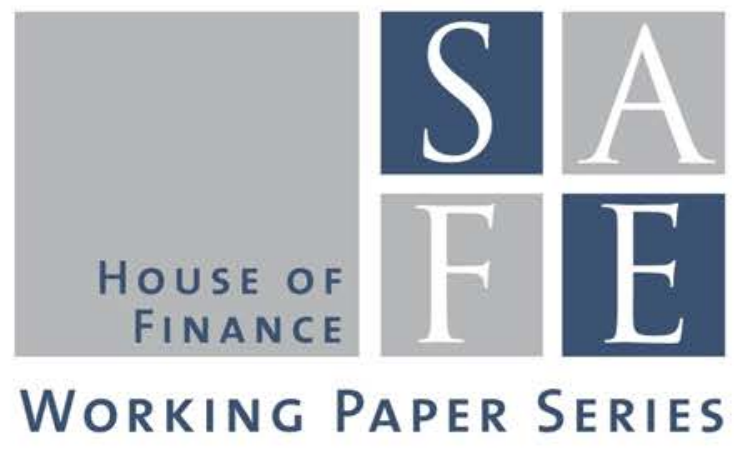

Holger Kraft - Farina Weiss

\title{
Consumption-Portfolio Choice with Preferences for Cash
}

SAFE Working Paper No. 181

SAFE I Sustainable Architecture for Finance in Europe A cooperation of the Center for Financial Studies and Goethe University Frankfurt 


\section{Non-Technical Summary}

Following the classical work by Merton $(1969,1971)$, most papers that study consumptionportfolio decisions disregard money. However, as for instance pointed out by Obstfeld and Rogo (1996), p. 513, "many of the most intriguing and important questions in international finance involve money". Now, there are several ways to take money into account. One approach assumes that money enters the utility function. This idea of modeling preferences for liquidity is well established. Starting with Sidrauski (1967) and Brock (1974), a strand of macroeconomic literature uses "money in the utility function" to address economic problems involving monetary issues. Intuitively, this approach could be justified by the implicit assumption that the agent has utility from both consumption and leisure. Real money balance thus enters the utility function indirectly since agents save time in conducting their transactions when holding cash. Despite its widespread use in the theory of monetary policy, a rigorous treatment of consumption-portfolio decisions with preferences for cash is missing. Our paper fills this gap and studies a canonical consumption-portfolio problem of a household with these preferences. We provide closed-form solutions in both the finite- and infinite-horizon case and also prove formal verification theorems showing that these solutions are indeed optimal.

We find that in the finite-horizon setting preferences for cash lead to time-dependent risky portfolio shares that are decreasing in age for reasonable calibrations. Such a pattern is qualitatively in line with rules of thumb that the risky share should decrease in age. It is also supported by recent empirical evidence showing that households who participate in the stock market have high and fairly constant risky shares during young ages, while investors reduce their risky share at a steady pace from about age 45 until they reach retirement (see Fagereng, Gottlieb, and Guiso (2017)). 


\title{
Consumption-Portfolio Choice with Preferences for Cash
}

This version: September 4, 2017

\author{
Holger Kraft \\ Goethe University, Faculty of Economics and Business Administration, \\ Frankfurt am Main, Germany, \\ email: holgerkraft@finance.uni-frankfurt.de
}

\section{Farina Weiss}

Goethe University, Faculty of Economics and Business Administration, Frankfurt am Main, Germany, email: weiss@safe.uni-frankfurt.de

ACKNOWLEDGEMents: Holger Kraft gratefully acknowledges financial support by Deutsche Forschungsgemeinschaft (DFG) as well as the Center of Excellence SAFE, funded by the State of Hessen initiative for research LOEWE. Farina Weiss gratefully acknowledges financial support by the Center of Excellence SAFE, funded by the State of Hessen initiative for research LOEWE. 


\section{Consumption-Portfolio Choice with Preferences for Cash}

ABstRaCT: This paper studies a consumption-portfolio problem where money enters the agent's utility function. We solve the corresponding Hamilton-JacobiBellman equation and provide closed-form solutions for the optimal consumption and portfolio strategy both in an infinite- and finite-horizon setting. For the infinite-horizon problem, the optimal stock demand is one particular root of a polynomial. In the finite-horizon case, the optimal stock demand is given by the inverse of the solution to an ordinary differential equation that can be solved explicitly. We also prove verification results showing that the solution to the Bellman equation is indeed the value function of the problem. From an economic point of view, we find that in the finite-horizon case the optimal stock demand is typically decreasing in age, which is in line with rules of thumb given by financial advisers and also with recent empirical evidence.

KEYWORDS: consumption-portfolio choice, money in the utility function, stock demand, stochastic control

JEL-Classification: G11, C61 


\section{Introduction}

Following the classical work by Merton (1969, 1971), most papers that study consumptionportfolio decisions disregard money. However, as for instance pointed out by Obstfeld and Rogoff (1996), p. 513, "many of the most intriguing and important questions in international finance involve money". Now, there are several ways to take money into account. One approach assumes that money enters the utility function. This idea of modeling preferences for liquidity is well established. Starting with Sidrauski (1967) and Brock (1974), a strand of macroeconomic literature uses "money in the utility function" to address economic problems involving monetary issues. ${ }^{1}$ Recent papers in financial economics applying this approach involve Balvers and Huang (2009) and Gu and Huang (2013), among others. Intuitively, this approach could be justified by the implicit assumption that the agent has utility from both consumption and leisure. Real money balance thus enters the utility function indirectly since agents save time in conducting their transactions when holding cash.

Despite its widespread use in the theory of monetary policy, a rigorous treatment of consumptionportfolio decisions with preferences for cash is missing. Our paper fills this gap and studies a canonical consumption-portfolio problem of a household with these preferences. We provide closed-form solutions in both the finite- and infinite-horizon case and also prove formal verification theorems showing that these solutions are indeed optimal. For the infinite-horizon case, the optimal stock demand is characterized by a polynomial. More precisely, it is one particular root of this polynomial. For the finite-horizon case, we show that the optimal stock demand is given by the inverse of the solution to an ordinary differential equation. The solution to the differential equation can be calculated explicitly. We also find that the relative location of the terminal condition for this differential equation and the solution to the infinite-horizon case determines whether the optimal stock demand is increasing or decreasing over time. Since individual optimality of household decisions is part of every general equilibrium analysis, our analysis also contributes to this field.

From an economic point of view, we find that in the finite-horizon setting preferences for cash lead to time-dependent risky portfolio shares that are decreasing in age for reasonable calibrations. Such a pattern is qualitatively in line with rules of thumb that the risky share should decrease in age. It is also supported by recent empirical evidence showing that households who participate in the stock market have high and fairly constant risky shares during young ages,

\footnotetext{
${ }^{1}$ See, e.g., Obstfeld and Rogoff (1996), p. 530, for more details and references. Google Scholar reports more than 1,600 citations for Sidrauski (1967) and more than 600 for Brock (1974).
} 
while investors reduce their risky share at a steady pace from about age 45 until they reach retirement (see Fagereng, Gottlieb, and Guiso (2017)).

Our paper is related to Dixit and Goldman (1970) who also assume that money enters the agent's utility. However, they consider a discrete-time setup and analyze first-order conditions only. In contrast to our paper, they cannot solve the model further and do not provide closedform solutions or a verification theorem. Other related papers that assume that money provides utility are Fama and Farber (1979) and LeRoy (1984a,b). Fama and Farber (1979) study an economy where the government provides money supply in a partial equilibrium model while LeRoy (1984a) and LeRoy (1984b) consider a similar general equilibrium setup. An alternative way to derive a demand for money is the cash-in-advance approach. In this approach, money is necessary to make transactions and does not directly affects the utility (see, e.g., Lucas (1982) and Svensson (1985)).

The remainder of the paper is structured as follows: Section 2 describes our framework. Section 3 provides and solves the Hamilton-Jacobi-Bellman equation of the agent's consumptionportfolio problem. It also studies some properties of the first-order conditions. Sections 4 and 5 analyze the infinite- and finite-horizon case in detail and derive the candidates for the optimal controls. Section 6 shows that these candidates are indeed optimal and proves the corresponding verification results. Section 7 provides numerical examples. Section 8 concludes. The proofs of the verification results can be found in the Appendix.

\section{Framework}

We consider a canonical portfolio problem where the agent can hold cash or invest in a stock index (short: stock). The dynamics are given by ${ }^{2}$

$$
\begin{aligned}
d M & =M r d t \\
d S & =S[\mu d t+\sigma d W] .
\end{aligned}
$$

The agent's wealth satisfies the dynamic budget constraint

$$
d X=X[(r+\pi \eta) d t+\sigma \pi d W]-c d t
$$

where $\eta=\mu-r$ and $\sigma>0$ are the stock's excess return and volatility, $\pi$ is the proportion invested in stock, and $c$ denotes consumption. The agent maximizes expected utility from

\footnotetext{
${ }^{2}$ For notational convenience, we omit time indices whenever it is not necessary for clarity.
} 
consumption and terminal wealth, but has also preferences for holding cash. This is because cash balances, by virtue of their liquidity, provide services (see, e.g., Obstfeld and Rogoff (1996) and the references therein). Therefore, the agent maximizes

$$
\sup _{\pi, c} \int_{0}^{T} e^{-\delta t} \mathrm{E}\left[u\left(c_{t}, X_{t}\left(1-\pi_{t}\right)\right)\right] d t+e^{-\delta T} \mathrm{E}\left[U\left(X_{T}, X_{T}\left(1-\pi_{T}\right)\right)\right] .
$$

Notice that $X_{t}\left(1-\pi_{t}\right)$ equals the amount invested in cash at time $t$. We assume that the agent's utility functions are given by

$$
u(c, \ell)=\frac{1}{1-\gamma}\left(c^{\beta} \ell^{1-\beta}\right)^{1-\gamma}, \quad U(x, \ell)=\frac{1}{1-\gamma}\left(x^{\alpha} \ell^{1-\alpha}\right)^{1-\gamma},
$$

i.e. he has a power utility function where the trade-off between consumption $c$ or wealth $x$ and liquid funds $\ell$ is measured by a Cobb-Douglas function. The constants $\alpha, \beta \in(0,1]$ can be interpreted as weights. For $\alpha=1$, we obtain standard bequest $U(x)=x^{1-\gamma} /(1-\gamma)$ as a special case. Throughout, we make the standing assumption that the risk aversion coefficient satisfies

$$
\gamma>1
$$

This requirement is imposed since risk aversions below one are not reasonable from an economic point of view. ${ }^{3}$ Besides, we assume that the agent's optimal stock demand in an ordinary Merton (1969, 1971) problem were positive, i.e. ${ }^{4}$

$$
m \equiv \frac{\eta}{\gamma \sigma^{2}}>0
$$

From an economic point of view, this assumption is not restrictive. The condition $m \geq 0$ is only violated if the expected excess return is negative, which is unrealistic and not in line with empirical estimates for major stock markets (e.g. US stock market). The case $m=0$ is trivial in our setting since even without preferences for cash the agent invests all his funds in the money market account. Therefore, preferences for cash have no effect on his portfolio decisions in this case. Finally, for simplicity we assume that

$$
r \geq-\frac{\delta}{\gamma-1}
$$

which is always satisfied for positive interest rates and can only be violated if the interest rate is sufficiently negative. ${ }^{5}$

\footnotetext{
${ }^{3}$ See, e.g., Munk (2013), pp. $175 \mathrm{ff}$.

${ }^{4}$ Table 1 summarizes all relevant constants that are defined in this paper.

${ }^{5}$ Actually, condition (2.4) could be relaxed further. This condition is sufficient to ensure that $D>0$ (see equation (4.31)). Instead we could also impose the condition that $D$ is positive.
} 


\section{Bellman Equation}

The Hamilton-Jacobi-Bellman equation (short: Bellman equation) for our problem is given by

$$
0=\sup _{\pi, c}\left\{G_{t}+x r G_{x}+x \pi \eta G_{x}-c G_{x}+0.5 x^{2} \sigma^{2} \pi^{2} G_{x x}-\delta G+u(c, x(1-\pi))\right\}
$$

with terminal condition $G(T, x)=U(x, x(1-\pi(T)))$. The first-order conditions become

$$
\begin{aligned}
G_{x} & =\left(c^{\beta}(x(1-\pi))^{1-\beta}\right)^{-\gamma} \beta c^{\beta-1}(x(1-\pi))^{1-\beta} \\
x \eta G_{x}+x^{2} \sigma^{2} \pi G_{x x} & =\left(c^{\beta}(x(1-\pi))^{1-\beta}\right)^{-\gamma}(1-\beta) c^{\beta}(x(1-\pi))^{-\beta} x .
\end{aligned}
$$

Rewriting (3.6) as

$$
G_{x}=\beta c^{\beta(1-\gamma)-1}(x(1-\pi))^{(1-\beta)(1-\gamma)}
$$

the first-order condition (3.7) becomes

$$
x \eta G_{x}+x^{2} \sigma^{2} \pi G_{x x}=\frac{1-\beta}{\beta} \frac{1}{1-\pi} c G_{x}
$$

or

$$
\pi=\left(\frac{\eta}{\sigma^{2}}-\frac{1-\beta}{\beta} \frac{1}{1-\pi} \frac{c}{x}\right) \frac{-G_{x}}{x G_{x x}}
$$

or, alternatively,

$$
c=\frac{\beta}{1-\beta}\left(x(1-\pi) \eta+\sigma^{2} \pi(1-\pi) \frac{x^{2} G_{x x}}{G_{x}}\right) .
$$

Besides, solving (3.6) for optimal consumption yields

$$
\begin{aligned}
c & =\beta^{\frac{1}{1-\beta(1-\gamma)}}(x(1-\pi))^{\frac{(\beta-1)(1-\gamma)}{\beta(1-\gamma)-1}} G_{x}^{\frac{1}{\beta(1-\gamma)-1}} \\
& =\beta^{\frac{1}{\kappa}}(x(1-\pi))^{\frac{(\beta-1)(\gamma-1)}{\kappa}} G_{x}^{-\frac{1}{\kappa}}
\end{aligned}
$$

where $\kappa \equiv 1+\beta(\gamma-1)>0$. Notice that by setting $\beta=1$ we can recover the standard results of models without preferences for cash. Using the above relations, we can calculate some terms in the Bellman equation more explicitly

$$
\begin{aligned}
c G_{x} & =\beta^{\frac{1}{\kappa}}(x(1-\pi))^{\frac{(\beta-1)(\gamma-1)}{\kappa}} G_{x}^{\frac{\beta(\gamma-1)}{\kappa}} \\
u(c, x(1-\pi)) & =\frac{1}{1-\gamma} \beta^{\frac{\beta(1-\gamma)}{\kappa}}(x(1-\pi))^{\frac{(\beta-1)(\gamma-1)}{\kappa}} G_{x}^{\frac{\beta(\gamma-1)}{\kappa}} .
\end{aligned}
$$

Using these results we can eliminate $c$ in the Bellman equation:

$$
0=\sup _{\pi}\left\{G_{t}+x r G_{x}+x \pi \eta G_{x}+0.5 x^{2} \sigma^{2} \pi^{2} G_{x x}-\delta G+K(x(1-\pi))^{\frac{(\beta-1)(\gamma-1)}{\kappa}} G_{x}^{\frac{\beta(\gamma-1)}{\kappa}}\right\}
$$


with

$$
K \equiv \frac{1}{1-\gamma} \beta^{\frac{\beta(1-\gamma)}{\kappa}}-\beta^{\frac{1}{\kappa}}=\beta^{\frac{1}{\kappa}-1}\left(\frac{1}{1-\gamma}-\beta\right)=\beta^{\frac{1}{\kappa}-1} \frac{\kappa}{1-\gamma}<0 .
$$

By (3.11), the first-order condition for the optimal stock demand (3.8) becomes

$$
x \eta G_{x}+x^{2} \sigma^{2} \pi G_{x x}=\frac{1-\beta}{\beta} \frac{1}{1-\pi} \beta^{\frac{1}{\kappa}}(x(1-\pi))^{\frac{(\beta-1)(\gamma-1)}{\kappa}} G_{x}^{\frac{\beta(\gamma-1)}{\kappa}}
$$

or

$$
\eta+\sigma^{2} \pi \frac{x G_{x x}}{G_{x}}=\frac{1-\beta}{\beta} \beta^{\frac{1}{\kappa}}\left((1-\pi)^{\gamma} x^{\gamma} G_{x}\right)^{-\frac{1}{\kappa}} .
$$

We now conjecture

$$
G(t, x)=\frac{1}{1-\gamma} x^{1-\gamma} f(t)^{k}
$$

with a constant $k$ that will be chosen later on. The terminal condition on $f$ is given by

$$
f(T)^{k}=(1-\pi(T))^{(1-\alpha)(1-\gamma)} .
$$

Then the first-order condition (3.6) for consumption becomes

$$
\frac{c}{x}=\beta^{\frac{1}{\kappa}}\left((1-\pi)^{(1-\beta)(\gamma-1)} f^{k}\right)^{-\frac{1}{\kappa}}
$$

and the first-order condition (3.12) for stock

$$
\eta-\gamma \sigma^{2} \pi=\frac{1-\beta}{\beta} \beta^{\frac{1}{\kappa}}\left((1-\pi)^{\gamma} f^{k}\right)^{-\frac{1}{\kappa}} .
$$

For the moment we make the assumption that the following conditions hold. A formal verification proof that these assumptions are actually satisfied can be found in Section 6 .

(i) The consumption-portfolio problem has a smooth value function with $f \in C^{1}, f>0$, and $k \in \mathbb{R}$.

(ii) The FOCs (3.15) and (3.16) determine the optimal consumption and stock demand.

(iii) If the horizon is infinite, then a suitable transversality condition holds. ${ }^{6}$

Under these assumptions, the optimal stock demand characterized by (3.16) is deterministic and independent of the agent's wealth. Notice that (3.16) is of the form

$$
\eta-\gamma \sigma^{2} \pi(t)=h(t)
$$

with a strictly positive deterministic function $h(t)$.

The following proposition establishes global concavity of our problem:

\footnotetext{
${ }^{6}$ See, e.g., Duffie (2001).
} 
Proposition 3.1 (Concavity and Global Optimality). Assume that (i) holds. Then the function

$$
H(c, \pi) \equiv x \pi \eta G_{x}-c G_{x}+0.5 x^{2} \sigma^{2} \pi^{2} G_{x x}+u(c, x(1-\pi))
$$

is strictly concave, i.e. a solution to the FOCs (3.15) and (3.16) is a global maximum.

Proof. The sufficient conditions for a maximum are

$$
H_{c c}<0, \quad H_{\pi \pi}<0, \quad \operatorname{det}\left[\begin{array}{ll}
H_{c c} & H_{c \pi} \\
H_{\pi c} & H_{\pi \pi}
\end{array}\right]>0
$$

with

$$
\begin{aligned}
H_{c c} & =u_{c c}=\beta(\beta(1-\gamma)-1) c^{\beta(1-\gamma)-2}(x(1-\pi))^{(1-\beta)(1-\gamma)}<0, \\
H_{\pi \pi} & =-\gamma x^{1-\gamma} \sigma^{2} f^{k}+(1-\beta)[(1-\beta)(1-\gamma)-1] c^{\beta(1-\gamma)}(x(1-\pi))^{(1-\beta)(1-\gamma)-2} x^{2}<0, \\
H_{c \pi} & =H_{\pi c}=\beta(1-\beta)(1-\gamma) c^{\beta(1-\gamma)-1}(x(1-\pi))^{(1-\beta)(1-\gamma)-1}(-x) .
\end{aligned}
$$

Hence,

$$
\begin{aligned}
\operatorname{det}\left[\begin{array}{ll}
H_{c c} & H_{c \pi} \\
H_{\pi c} & H_{\pi \pi}
\end{array}\right] & =-\gamma x^{1-\gamma} \sigma^{2} f^{k} \beta[\beta(1-\gamma)-1] c^{\beta(1-\gamma)-2}(x(1-\pi))^{(1-\beta)(1-\gamma)} x^{2} \\
& +\beta(1-\beta)[(1-\beta)(1-\gamma)-1][\beta(1-\gamma)-1] c^{2 \beta(1-\gamma)-2}(x(1-\pi))^{2(1-\beta)(1-\gamma)-2} x^{2} \\
& >0 .
\end{aligned}
$$

Now, we obtain the following intuitive result.

Proposition 3.2 (Size of the Optimal Stock Demand). Under assumptions (i)-(iii) and conditions (2.2)-(2.4) the agent's stock demand is bounded from above as follows:

$$
\pi^{*}(t)<\min \{m, 1\},
$$

where $m$ is the stock demand in a Merton problem defined in (2.3).

Proof. For $m \leq 1$ we obtain $\pi^{*}(t)<m$ since (3.17) holds. For $m>1$ the FOC (3.16) dictates $\pi^{*}(t)<1$.

Substituting the guess (3.13) into the Bellman equation yields

$$
0=\frac{k}{1-\gamma} f_{t}+\left(r-\frac{\delta}{1-\gamma}+\pi \eta-0.5 \gamma \sigma^{2} \pi^{2}\right) f+K(1-\pi)^{-\frac{(\beta-1)(1-\gamma)}{\kappa}} f^{1-\frac{k}{\kappa}},
$$


where we assume that the Bellman equation is evaluated at the optimal stock demand $\pi=$ $\pi^{*}$. Hence, the separation (3.13) works, but the first-order condition (3.16) and the Bellman equation (3.19) constitute a coupled system of equations for $f$ and $\pi^{*}$. Since (3.16) can be rewritten as

$$
(1-\pi)^{\frac{(1-\beta)(1-\gamma)}{\kappa}-1} f^{-\frac{k}{\kappa}}=\frac{\beta}{1-\beta} \beta^{-\frac{1}{\kappa}}\left(\eta-\gamma \sigma^{2} \pi\right),
$$

the Bellman equation can be expressed as

$$
0=\frac{k}{1-\gamma} f_{t}+\left(r-\frac{\delta}{1-\gamma}+\pi \eta-0.5 \gamma \sigma^{2} \pi^{2}+\widetilde{K}\left(\eta-\gamma \sigma^{2} \pi\right)(1-\pi)\right) f
$$

with

$$
\widetilde{K} \equiv K \frac{\beta}{1-\beta} \beta^{-\frac{1}{\kappa}}=\frac{\kappa}{(1-\gamma)(1-\beta)}
$$

By choosing $k$ as

$$
k=\kappa=1+\beta(\gamma-1),
$$

one can isolate $f$ in $(3.16)$ :

$$
f=\frac{\frac{1-\beta}{\beta} \beta^{\frac{1}{\kappa}}}{\left(\eta-\gamma \sigma^{2} \pi\right)(1-\pi)^{\frac{\gamma}{\kappa}}} .
$$

Notice that the optimal stock demand thus directly determines the value function and vice versa. Furthermore, we can substitute into (3.15) and rewrite the consumption-wealth ratio

$$
\frac{c}{x}=\frac{\beta}{1-\beta}\left(\eta-\gamma \sigma^{2} \pi\right)(1-\pi),
$$

which is inversely related to the stock demand.

Taking derivatives in (3.21) w.r.t. time $t$ and substituting back into the Bellman equation leads to an differential equation for $\pi$ : First, we differentiate (3.21) w.r.t. time $t$ and get

$$
\frac{d f}{d t}=f \frac{d \pi}{d t}\left\{\frac{\gamma}{\kappa}(1-\pi)^{-1}+\gamma \sigma^{2}\left(\eta-\gamma \sigma^{2} \pi\right)^{-1}\right\}
$$

where we express the derivative in terms of $f$ and the derivative of $\pi$. Substituting $f$ and $f_{t}$ into (3.20) and using the definition (2.3) yields the following ODE for $\pi$ :

$$
\frac{d \pi}{d t}=\frac{\gamma-1}{k} \frac{P_{2}(\pi)(1-\pi)(m-\pi)}{(1-\pi)+\frac{\gamma}{\kappa}(m-\pi)}
$$

where $P_{2}(\pi)$ is a second-order polynomial in $\pi$ :

$$
P_{2}(\pi)=r-\frac{\delta}{1-\gamma}+\pi \eta-0.5 \gamma \sigma^{2} \pi^{2}+\widetilde{K} \gamma \sigma^{2}(m-\pi)(1-\pi)
$$

This leads to the following result concerning the slope of the stock demand over time: 
Lemma 3.3 (Slope of the Stock Demand). Suppose that assumptions (i)-(iii) hold. If conditions (2.2)-(2.4) are satisfied, then (3.18) holds and thus the denominator in (3.23) is always positive, i.e.

$$
(1-\pi)+\frac{\gamma}{\kappa}(m-\pi)>0
$$

In particular, the sign of the polynomial $P_{2}$ determines the sign of the slope of $\pi$ over time, i.e.

$$
P_{2}(\pi)>0 \Longleftrightarrow \frac{d \pi}{d t}>0 \quad \text { and } \quad P_{2}(\pi)<0 \quad \Longleftrightarrow \quad \frac{d \pi}{d t}<0 .
$$

Since $f$ must satisfy the terminal condition (3.14), the terminal condition for $\pi$ can be calculated from (3.16) by solving for $\pi(T)$ in the following equation

$$
\begin{aligned}
\eta-\gamma \sigma^{2} \pi(T) & =\frac{1-\beta}{\beta} \beta^{\frac{1}{\kappa}}\left((1-\pi(T))^{\gamma} f(T)^{k}\right)^{-\frac{1}{\kappa}} \\
& =C(1-\pi(T))^{-\frac{\kappa_{\alpha}}{\kappa}}
\end{aligned}
$$

with $\kappa_{\alpha} \equiv 1+\alpha(\gamma-1)$ and

$$
C \equiv \frac{1-\beta}{\beta} \beta^{\frac{1}{\kappa}}
$$

There are two special cases where the terminal condition becomes explicit. The following proposition summarizes these cases.

Proposition 3.4 (Terminal Condition on $\pi$ ). (i) If the liquidity preferences are the same for consumption and bequest, i.e. $\beta=\alpha$, we obtain

$$
\pi^{*}(T)=\frac{m+1}{2}-\sqrt{\frac{(m-1)^{2}}{4}+\frac{C}{\gamma \sigma^{2}}}<\min \{m, 1\} .
$$

(ii) If $\eta=\gamma \sigma^{2}$, i.e. $m=1$, then

$$
\pi^{*}(T)=1-\left(\frac{C}{\gamma \sigma^{2}}\right)^{\frac{\kappa}{\kappa+\kappa \alpha}}
$$

Proof. (i) In this case $\kappa_{\alpha}=\kappa$ and thus equation (3.25) becomes quadratic:

$$
\pi(T)^{2}+a \pi(T)+b=0 \quad \text { with } \quad a \equiv-(m+1), \quad b \equiv m-\frac{1-\beta}{\beta} \beta^{\frac{1}{\kappa}} \frac{1}{\gamma \sigma^{2}} .
$$

This equation has two real solutions, but one root is bigger than $0.5(m+1)$ and thus violates Proposition 3.2. Now, $C>0$ and thus

$$
\pi^{*}(T)=\frac{m+1}{2}-\sqrt{\frac{(m-1)^{2}}{4}+\frac{C}{\gamma \sigma^{2}}}<\frac{m+1}{2}-\frac{|m-1|}{2}=\min \{m, 1\}
$$


Therefore, (3.27) is the correct solution and (i) follows.

(ii) follows since $\eta-\gamma \sigma^{2} \pi(T)=\gamma \sigma^{2}(1-\pi(T))$.

Now, we can solve the ODE (3.23) by applying separation of variables:

$$
\frac{k}{\gamma-1} \int \frac{(1-\pi)+\frac{\gamma}{\kappa}(m-\pi)}{P_{2}(\pi)(1-\pi)(m-\pi)} d \pi=t+\text { const }
$$

where const can be determined by using terminal condition (3.25) on $\pi$.

\section{Infinite Horizon}

We first study the infinite-horizon case. Then the ODE (3.23) simplifies to an algebraic equation for the optimal stock demand:

$$
0=\frac{\gamma-1}{k} \frac{P_{2}(\pi)(1-\pi)(m-\pi)}{(1-\pi)+\frac{\gamma}{\kappa}(m-\pi)} .
$$

Notice that the optimal stock demand and in turn the function $f$ is now simply a constant. Under assumptions (i)-(iii) the optimal demand is a root of the polynomial. Since we impose (2.2)-(2.4), there are two possible solutions and we have to identify the correct one. The polynomial can be rewritten as follows

$$
P_{2}(\pi)=\widehat{K}\left(\pi^{2}+p \pi+q\right)
$$

with

$$
\widehat{K} \equiv \gamma \sigma^{2}(\widetilde{K}-0.5)<0, \quad p \equiv \frac{\eta-\widetilde{K}\left(\eta+\gamma \sigma^{2}\right)}{\widehat{K}}, \quad q \equiv \frac{r-\frac{\delta}{1-\gamma}+\widetilde{K} \eta}{\widehat{K}},
$$

and thus the two candidate solutions are

$$
\pi_{01 / 02}=-0.5 p \pm \sqrt{D} \quad \text { with } \quad D \equiv p^{2} / 4-q .
$$

To characterize the solutions, we need some technical results that are summarized in the following lemma. To simplify notation, we set

$$
\chi \equiv-\widetilde{K}=\frac{\kappa}{(\gamma-1)(1-\beta)}>0 .
$$

Lemma 4.1. We get the following representations:

$$
\begin{aligned}
-0.5 p & =\frac{m+(1+m) \chi}{1+2 \chi} \\
D & =\frac{m^{2}}{1+2 \chi}+\left(\frac{\chi(1-m)}{1+2 \chi}\right)^{2}+\frac{\frac{m}{\eta}\left(r-\frac{\delta}{1-\gamma}\right)}{0.5(1+2 \chi)} \\
-0.5 p-m & =\frac{\chi(1-m)}{1+2 \chi}
\end{aligned}
$$


Now assume that conditions (2.2)-(2.4) are satisfied. Then

$$
-0.5 p \in\left[\min \left\{m, \frac{1+m}{2}\right\}, \max \left\{m, \frac{1+m}{2}\right\}\right] \quad \text { and } \quad D>0 .
$$

Furthermore, if $m \leq 1$ we get for the distance between $-0.5 p$ and $m$

$$
-0.5 p-m \in[0, \sqrt{D})
$$

If $m>1$ we obtain

$$
-0.5 p-\sqrt{D}<1
$$

Proof. The representations follow from lengthy calculations. Furthermore, the function $\psi(x)=$ $\frac{m+(1+m) \chi}{1+2 \chi}$ is monotonic increasing for $m \leq 1$ (decreasing for $m>1$ ), starting at $m$ for $\chi=0$, and having the limit $0.5(1+m)$ for $\chi \rightarrow \infty$. Besides, $D>0$ since $\chi>0$, and $r \geq-\delta /(\gamma-1)$. For $m \leq 1$ we get $-0.5 p-m \geq 0$ since (4.32) and $\chi>0$. Furthermore, $-0.5 p-m<\sqrt{D}$ since the middle term in the representation of $D$ is $(-0.5 p-m)^{2}$ and the first term is strictly positive. To show (4.34) for $m>1$, notice that

$$
(-0.5 p-1)^{2}=\frac{(m-1)^{2}(1+\chi)^{2}}{(1+2 \chi)^{2}} .
$$

On the other hand,

$$
D>\frac{m^{2}}{1+2 \chi}+\left(\frac{\chi(1-m)}{1+2 \chi}\right)^{2}=\frac{m^{2}(1+2 \chi)+\chi^{2}(1-m)^{2}}{(1+2 \chi)^{2}} .
$$

Now, the result follows since

$$
(m-1)^{2}(1+\chi)^{2}=(m-1)^{2}(1+2 \chi)+\chi^{2}(m-1)^{2}<m^{2}(1+2 \chi)+\chi^{2}(1-m)^{2} .
$$

Remark. Notice that $-0.5 p-m<\sqrt{D}$ if $m \in(0,1]$, but $-0.5 p-m=\sqrt{D}$ if $m=0$. As already pointed out, the latter case is not interesting since then the agent puts all his wealth into the money market account in the standard portfolio problem anyway. Therefore, liquidity preferences are irrelevant for the portfolio decision. This trivial case is ruled out by assumption (2.3).

Now, we can characterize the optimal stock demand as follows:

Proposition 4.2 (Optimal Stock Demand for an Infinite Horizon). Under assumptions (i)-(iii) and conditions (2.2)-(2.4) the optimal stock demand for an infinite horizon is given by

$$
\pi^{\infty} \equiv \pi_{02}=-0.5 p-\sqrt{D}<\min \{m, 1\} .
$$


Proof. For the first root, we get $\pi_{01}=-0.5 p+\sqrt{D}>\min \left\{m, \frac{1+m}{2}\right\}$, which violates the result of Proposition 3.2. Since (4.33) or (4.34), respectively, $\pi_{02}$ is the only relevant solution to the first-order condition.

Remark. Given our previous results the polynomial of degree four in the numerator on the right-hand side of (3.23) has four real-valued roots:

$$
\pi_{01}>\min \left\{m, \frac{1+m}{2}\right\}, \quad \pi^{\infty}<\min \{m, 1\}, \quad \pi_{03}=1, \quad \pi_{04}=m,
$$

where the last two become a double root for $m=1$. It can thus be written as

$$
P_{2}(\pi)(1-\pi)(m-\pi)=\widehat{K}\left(\pi_{01}-\pi\right)\left(\pi^{\infty}-\pi\right)(1-\pi)(m-\pi) .
$$

For $m=1$ the representations in (4.30) become

$$
p=-2, \quad q=1+\frac{r-\frac{\delta}{1-\gamma}+0.5 \gamma \sigma^{2}}{\widehat{K}} \quad \Longrightarrow \quad D=\frac{(\widetilde{r}+1)(\gamma-1)(1-\beta)}{\gamma}>0
$$

with $\widetilde{r} \equiv 2\left(r-\frac{\delta}{1-\gamma}\right) /\left(\gamma \sigma^{2}\right)$. Therefore,

$$
\pi_{01}=1+\sqrt{D}, \quad \pi_{02}=1-\sqrt{D} .
$$

This leads to the following result:

Corollary 4.3 (Optimal Stock Demand for an Infinite Horizon and $m=1$ ). Under assumptions (i)-(iii) and conditions (2.2)-(2.4) the optimal stock demand for an infinite horizon and $m=1$ is given by

$$
\pi^{\infty}=\pi_{02}=1-\sqrt{D} .
$$

Since the optimal demand in an ordinary Merton problem is one, we now get the clean result that the adjustment for liquidity preferences is given by $\sqrt{D}$.

\section{Finite Horizon}

We first study the finite-horizon case for $m \neq 1$. The case $m=1$ is analyzed below. Furthermore, notice that if $\pi^{*}(T)=\pi^{\infty}$, then the optimal stock demand is constant and equal to $\pi^{*}(t)=\pi^{\infty}$. This follows directly from (3.23). We thus impose

$$
\pi^{*}(T) \neq \pi^{\infty}
$$

Throughout this section, we also assume that assumptions (i)-(iii) and conditions (2.2)-(2.4) are satisfied. 


\subsection{The Case $m \neq 1$}

Given our previous results we can rewrite (3.28):

$$
\mathcal{G}(\pi) \equiv \int \frac{(1-\pi)+\frac{\gamma}{\kappa}(m-\pi)}{\left(\pi_{01}-\pi\right)\left(\pi^{\infty}-\pi\right)(1-\pi)(m-\pi)} d \pi=-\bar{K} t+\text { const }
$$

with $\bar{K} \equiv-\widehat{K} \frac{\gamma-1}{k}>0$. The following lemma summarizes some relevant properties of the function $\mathcal{G}$.

Lemma 5.1 (Properties of $\mathcal{G}$ ). (i) The mapping $\mathcal{G}$ is a well-defined real-valued function on $(-\infty, \min \{m, 1\}) \backslash\left\{\pi^{\infty}\right\}$ with the representation

$$
\mathcal{G}(\pi) \equiv-\alpha_{1} \ln \left(\pi_{01}-\pi\right)-\alpha_{2} \ln \left|\pi^{\infty}-\pi\right|-\alpha_{3} \ln (1-\pi)+\alpha_{4} \ln (m-\pi)
$$

where

$$
\begin{array}{rlrl}
\alpha_{1} & \equiv \frac{1+\frac{\gamma}{k} m-\pi_{01}\left(1+\frac{\gamma}{k}\right)}{\left(\pi_{01}-\pi^{\infty}\right)\left(1-\pi_{01}\right)\left(\pi_{01}-m\right)}, & \alpha_{2} & \equiv \frac{1+\frac{\gamma}{k} m-\pi^{\infty}\left(1+\frac{\gamma}{k}\right)}{\left(\pi_{01}-\pi^{\infty}\right)\left(1-\pi^{\infty}\right)\left(m-\pi^{\infty}\right)}, \\
\alpha_{3} \equiv \frac{\gamma}{\left(1-\pi_{01}\right)\left(1-\pi^{\infty}\right)}, & \alpha_{4} \equiv \frac{1}{\left(\pi_{01}-m\right)\left(m-\pi^{\infty}\right)} .
\end{array}
$$

(ii) For all $m>0$, we have $\alpha_{2}>0$. If $m \in(0,1]$, then $\alpha_{4}>0$. If $m>1$, then $\alpha_{3}<0$.

(iii) $\mathcal{G}(\pi) \rightarrow \infty$ if $\pi \searrow \pi^{\infty}$ or $\pi \nearrow \pi^{\infty}$.

(iv) If $m \in(0,1]$, then $\mathcal{G}(\pi) \rightarrow-\infty$ for $\pi \nearrow m$. If $m>1$, then $\mathcal{G}(\pi) \rightarrow-\infty$ for $\pi \nearrow 1$.

(v) $\mathcal{G}^{\prime}(\pi)>0$ for $\pi<\pi^{\infty}$ and $\mathcal{G}^{\prime}(\pi)<0$ for $\pi \in\left(\pi^{\infty}, m\right]$. In particular, $\mathcal{G}$ has a well-defined inverse $\mathcal{G}^{-1}$ for $\pi<\pi^{\infty}$ and for $\pi \in\left(\pi^{\infty}, m\right]$.

Proof. (i) follows from integrating the partial fraction expansion. (ii) follows from (4.35). (iii) and (iv) follow from the representation of $\mathcal{G}$ and (ii). Notice that for $m \in(0,1)$ the terms involving $\alpha_{1}$ and $\alpha_{3}$ are bounded, whereas for $m>1$ the terms involving $\alpha_{1}$ and $\alpha_{4}$ are bounded. Finally, by construction, we have

$$
\mathcal{G}^{\prime}(\pi)=\frac{(1-\pi)+\frac{\gamma}{\kappa}(m-\pi)}{\left(\pi_{01}-\pi\right)\left(\pi^{\infty}-\pi\right)(1-\pi)(m-\pi)} .
$$

The sign of $\mathcal{G}^{\prime}$ is determined by the term $\left(\pi^{\infty}-\pi\right)$ since all other terms are positive and thus (v) follows.

The constant in (5.38) is given by $\pi^{*}(T)$, which can be determined by (3.25). Therefore, we obtain

$$
\text { const }=\mathcal{G}\left(\pi^{*}(T)\right)+\bar{K} T \quad \Longrightarrow \quad \mathcal{G}\left(\pi^{*}(t)\right)=\bar{K}(T-t)+\mathcal{G}\left(\pi^{*}(T)\right)
$$


where $\mathcal{G}\left(\pi^{*}(T)\right)$ can be calculated by substituting $\pi^{*}(T)$ into (5.39). The optimal demand $\pi^{*}$ must be smooth since it is directly related to $f$, which must be a $C^{1}$-function. Therefore, the properties of $\mathcal{G}$ imply the following result:

Proposition 5.2 (Size and Slope of the Stock Demand). (i) If $\pi^{*}(T)<\pi^{\infty}$, then $\pi^{*}(t)<$ $\pi^{\infty}$ and $d \pi^{*}(t) / d t<0$. (ii) If $\pi^{*}(T) \in\left(\pi^{\infty}, \min \{m, 1\}\right)$, then $\pi^{*}(t) \in\left(\pi^{\infty}, \min \{m, 1\}\right)$ and $d \pi^{*}(t) / d t>0$. In any case, if (5.37) holds, then the optimal stock demand is given by

$$
\pi^{*}(t)=\mathcal{G}^{-1}\left(\bar{K}(T-t)+\mathcal{G}\left(\pi^{*}(T)\right)\right)
$$

In particular, $\pi^{*}$ is a smooth $C^{1}$-function, which by (3.21) is also true for $f$.

Proof. Notice that

$$
\frac{d \pi^{*}(t)}{d t}=\frac{d \mathcal{G}^{-1}}{d t}(\ldots) \cdot(-\bar{K})
$$

Since $\bar{K}>0$, the result follows by Lemma 5.1 (iv). Besides, $\pi^{*}$ is well-defined because of Lemma $5.1(\mathrm{v})$.

Remark. Figure 1 that is discussed in Section 7 shows an example of the function $\mathcal{G}$ for the benchmark calibration reported in Table 2 .

As we will see in our numerical examples, the first case with $\pi^{*}(T)<\pi^{\infty}$ is the more natural situation, whereas the second case occurs less frequently (e.g. for unrealistically high risk aversions). From an economic point of view, the first case generates an interesting stock demand that is falling over the life-cycle. So adding preferences for liquidity to a standard Merton framework can generate a decreasing stock demand.

\subsection{The Case $m=1$}

For the case $m=1$ we have $\eta=\gamma \sigma^{2}$ and thus equation (5.38) simplifies into

$$
-\frac{2(1-\beta)}{\gamma \sigma^{2}} \int \frac{1}{\left(\pi_{01}-\pi\right)\left(\pi^{\infty}-\pi\right)(1-\pi)} d \pi=t+\text { const }
$$

because $-\left(1+\frac{\gamma}{\kappa}\right) / \bar{K}=-2(1-\beta) /\left(\gamma \sigma^{2}\right)$. Since

$$
\int \frac{1}{\left(\pi_{01}-\pi\right)\left(\pi^{\infty}-\pi\right)(1-\pi)} d \pi=\frac{2 \ln (1-\pi)-\ln \left|\pi^{\infty}-\pi\right|-\ln \left(\pi_{01}-\pi\right)}{2 D}
$$

we can rewrite (5.41) as follows

$$
\mathcal{H}\left(\pi^{*}(t)\right)=\mathcal{H}\left(\pi^{*}(T)\right) e^{\frac{D \gamma \sigma^{2}}{1-\beta}(T-t)},
$$


where $^{7}$

$$
\mathcal{H}(\pi) \equiv \frac{(1-\pi)^{2}}{\left|\pi^{\infty}-\pi\right|\left(\pi_{01}-\pi\right)}=\frac{(1-\pi)^{2}}{|1-\sqrt{D}-\pi|(1+\sqrt{D}-\pi)}, \quad \pi \in\left(-\infty, \pi^{\infty}\right) \cup\left(\pi^{\infty}, 1\right),
$$

and $D$ is given in (4.36). The constant $\mathcal{H}\left(\pi^{*}(T)\right)$ reads

$$
\mathcal{H}\left(\pi^{*}(T)\right)=\frac{\left(\frac{C}{\gamma \sigma^{2}}\right)^{\frac{2 \kappa}{\kappa+\kappa \alpha}}}{\left|D-\left(\frac{C}{\gamma \sigma^{2}}\right)^{\frac{2 \kappa}{\kappa+\kappa \alpha}}\right|},
$$

where $C$ is given by (3.26). In the special case $\alpha=\beta$, this simplifies further

$$
\mathcal{H}\left(\pi^{*}(T)\right)=\frac{C}{\left|D \gamma \sigma^{2}-C\right|}
$$

Again it is not a priori clear whether $\pi^{*}(T)>\pi^{\infty}$ or $\pi^{*}(T)<\pi^{\infty}$. We can now invert the function $\mathcal{H}$ :

Lemma 5.3 (Inverse of $\mathcal{H}$ ). (i) If $\pi \in\left(-\infty, \pi^{\infty}\right)$, the inverse of $\mathcal{H}$ is given by

$$
\mathcal{H}^{-1}(y)=1-\sqrt{\frac{D y}{y-1}}, \quad y>1 \text {. }
$$

(ii) If $\pi \in\left(\pi^{\infty}, 1\right)$, the inverse of $\mathcal{H}$ is given by

$$
\mathcal{H}^{-1}(y)=1-\sqrt{\frac{D y}{y+1}}
$$

Proof. (i) In this case, we must invert the function

$$
\mathcal{H}(\pi)=\frac{(1-\pi)^{2}}{(1-\sqrt{D}-\pi)(1+\sqrt{D}-\pi)} .
$$

This yields two candidates

$$
\mathcal{H}^{-1}(y)=1 \pm \sqrt{\frac{D y}{y-1}}, \quad y>1 \text {. }
$$

We must have $\mathcal{H}^{-1}(y) \leq 1$, since $\pi^{*} \leq 1$. Therefore, (5.44) follows. Notice that $y>1$ is not a restriction since the right-hand side of (5.42) is strictly greater than one. This is because $\mathcal{H}$ as a function of $\sqrt{D}$ is one for $\sqrt{D}=0$, which is excluded by our assumptions, and strictly increasing in $\sqrt{D}$ for $\sqrt{D}>0$. The case (ii) follows analogously to (i).

Consequently, we get a fully explicit representation of the optimal stock demand:

\footnotetext{
${ }^{7}$ The case $\pi=\pi^{\infty}$ is not relevant due to assumption (5.37).
} 
Proposition 5.4 (Optimal Stock Demand for $m=1$ ). (i) If $\pi^{*}(T) \in\left(-\infty, \pi^{\infty}\right)$, the optimal stock demand is explicitly given by

$$
\pi^{*}(t)=1-\sqrt{\frac{D}{1-e^{-\frac{D \gamma \sigma^{2}}{1-\beta}(T-t)} / \mathcal{H}\left(\pi^{*}(T)\right)}} .
$$

(ii) If $\pi^{*}(T) \in\left(\pi^{\infty}, 1\right)$, the optimal stock demand is explicitly given by

$$
\pi^{*}(t)=1-\sqrt{\frac{D}{1+e^{-\frac{D \gamma \sigma^{2}}{1-\beta}(T-t)} / \mathcal{H}\left(\pi^{*}(T)\right)}} .
$$

In both cases, the constant $\mathcal{H}\left(\pi^{*}(T)\right)$ is given by (5.43).

Proof. follows from (5.42) and Lemma 5.3.

Remarks. a) If the horizon goes to infinity, i.e. $T \rightarrow \infty$, we recover the result of Corollary 4.3.

b) By construction of the inverse, the right-hand side in both cases becomes $\pi^{*}(T)$ if $t=T$.

c) By differentiating $\pi^{*}(t)$ w.r.t. time $t$, one can check that for (i) the stock demand is increasing over time, whereas it is decreasing for (ii), which is in line with our previous results.

\section{Verification}

We now prove that the assumptions (i)-(iii) imposed in Section 3 are satisfied for our problem, i.e. the candidate $G$ is indeed the value function and $\left(\pi^{*}, c^{*}\right)$ is the optimal strategy. Notice that we will sometimes use the notation $X^{\pi, c}$ to emphasize that the wealth dynamics depend on the controls $\pi$ and $c$. First, we need to define the set of admissible strategies.

Definition 6.1 (Admissible Strategy). A strategy $(\pi, c)$ is said to be admissible if the following conditions are satisfied:

(i) The processes $\pi$ and $c$ are progressively measurable w.r.t. the filtration generated by the Brownian motion $W$,

(ii) for all initial conditions $\left(t_{0}, x_{0}\right) \in[0, T] \times(0, \infty)$ the wealth equation (2.1) with $X^{\pi, c}\left(t_{0}\right)=x_{0}$ has a pathwise unique solution $\left\{X_{t}^{\pi, c}\right\}_{t \in\left[t_{0}, T\right]}$,

(iii) $X^{\pi, c} \geq 0$,

(iv) $c \geq 0$ and $\pi \leq 1$

(v) $c \leq \bar{c} X^{\pi, c}$ and $\pi \geq \underline{\pi}$ for constants $\bar{c}$ and $\underline{\pi}$ that can be different for different strategies.

We denote the set of admissible strategies by $\mathcal{A}$. 
Remarks. a) Notice that (i)-(iii) are for instance satisfied if $c$ and $\pi$ are smooth deterministic functions.

b) If the conditions in (iv) do not hold, then the utility functional is not well-defined.

c) The conditions in (v) are imposed to simplify matters. They could be relaxed by imposing suitable integrability conditions on $c$ and $\pi$. From an economic point of view, it seems however reasonable that the stock demand is bounded from below and that the consumption-wealth ratio is bounded from above.

First, we summarize our previous results concerning the candidates for the value function and the optimal controls.

Theorem 6.2 (Candidates for Value Function and Controls). Assume that conditions (2.2)(2.4) hold. Then we obtain the following: (i) The candidate (5.40) for the optimal stock demand $\pi^{*}$ is a well-defined real-valued $C^{1}$-function defined on $[0, T]$ and mapping into $\left(-\infty, \pi^{\infty}\right)$ if $\pi^{*}(T)<\pi^{\infty}$ and mapping into $\left(\pi^{\infty}, m\right)$ if $\pi^{*}(T)>\pi^{\infty}$.

(ii) The candidates $f$ and $c^{*}$ are well-defined real-valued $C^{1}$-functions given by (3.21) and (3.22).

(iii) The pair $\left(\pi^{*}, c^{*}\right)$ is admissible in the sense of Definition 6.1.

Proof. (i) follows from Proposition 5.2. (ii) follows from (3.21) and (3.22) since for our candidate $\pi^{*}$ we have $\pi^{*}<\min \{m, 1\}$. In particular, there exist a constant $\bar{c}$ such that $c^{*} \leq$ $\bar{c} X^{\pi^{*}, c^{*}}$. Since $\pi^{*}$ is a smooth deterministic function on $[0, T]$, one can check that the pair $\left(\pi^{*}, c^{*}\right)$ is admissible.

Now, we can verify that the candidates for the optimal stock demand and consumption are indeed optimal.

Theorem 6.3 (Verification for a Finite Horizon). The function G given by (3.13) is the value function of the problem and $\pi^{*}$ and $c^{*}$ are the optimal stock demand and consumption.

Proof. See Appendix A.

If the horizon is infinite, then the value function and the optimal strategy become timeindependent. In particular, $G$ satisfies the Bellman equation (3.5) where the time derivative $G_{t}$ is set to zero. The candidate for the optimal stock demand is the constant $\pi^{\infty}$ and satisfies the algebraic equation (4.29) that we have analyzed in detail in Section 4. In turn, the candidates for $f$ and $c / x$ that are still given by (3.21) and (3.22) are also constant. To ensure that our verification result provided in Theorem 6.3 carries over to the infinite-horizon case, we have 
to prove a so-called transversality result. 8 If this is satisfied, then loosely speaking beqeust becomes negligible if the horizon gets larger. Formally, we obtain the following result:

Theorem 6.4 (Verification for an Infinite Horizon). Assume that the candidate for the optimal stock demand satisfies $\pi^{\infty}>0$. Then $\pi^{\infty}$ is the optimal stock demand and the value function is given by

$$
G(x)=\frac{1}{1-\gamma} x^{1-\gamma} f^{k}
$$

where $f$ is a constant that is determined by $\pi^{\infty}$ via (3.21). The optimal consumption-wealth ratio $c^{\infty} / x$ is also constant and given by

$$
\frac{c^{\infty}}{x}=\frac{\beta}{1-\beta}\left(\eta-\gamma \sigma^{2} \pi^{\infty}\right)\left(1-\pi^{\infty}\right)
$$

Proof. See Appendix A.

Remark. The condition $\pi^{\infty}>0$ excludes pathological cases where the candidate for the optimal stock demand is negative. This can only occur if $\beta$ becomes very small.

\section{Numerical Examples}

To illustrate the effect of preferences for cash, we now consider the stock demands for a baseline calibration and also perform some comparative statics. Our calibration is summarized in Table 2. The values of the real interest rate $r$, the equity premium $\eta$, and the stock volatility $\sigma$ are in line with US data starting in 1960. As stock market data, we use returns on the CRSP value-weighted market portfolio inclusive of the NYSE, AMEX, and NASDAQ markets (cum dividend). The risk-free asset is estimated from the Treasury bill yield provided by the Risk Free File on CRSP Bond tape. To obtain real values, all time-series are deflated using the consumer price index (CPI) taken from the website of the Bureau of Labor Statistics. We slightly reduce the equity premium to $5 \%$ to account for survivorship bias (Brown, Goetzmann, and Ross (1995)) as well as the decline in discount rates and the implied unexpected capital gains over the sample period (Fama and French (2002)). The choices of the risk aversion $\gamma=3$ and time-preference rate $\delta=0.03$ are in line with the literature on life-cycle portfolio choice (see, e.g., Munk (2013) and the references therein), but we also vary the risk aversion $\gamma$ and the consumption weight $\beta$. We assume that $\alpha=1$, i.e. bequest does not involve preferences

\footnotetext{
${ }^{8}$ See, e.g., Duffie (2001), p. 213.
} 
for cash. However, choosing for instance $\alpha=\beta=0.99$ hardly changes our results. The time horizon is 50 years (e.g. age 25 until age 75 ).

We first illustrate how the function $\mathcal{G}$ given by (5.39) looks like. Recall that it plays an important role for the optimal stock demand. Figure 1 depicts $\mathcal{G}$ for our benchmark calibration. As we have seen in Section 5, the shape of of $\mathcal{G}$ is crucial for the size and slope of the optimal stock demand over time. In particular, the location of the pole of $\mathcal{G}$, which is the solution $\pi^{\infty}$ to the infinite-horizon case, relative to the terminal condition $\pi^{*}(T)$ determines whether the stock demand is increasing or decreasing. In our benchmark case, we get $\pi^{*}(T)=0.3867$, which is smaller than the optimal demand $\pi^{\infty}$ of the infinite-horizon problem. Therefore, by Proposition 5.2 , the left branch of the function $\mathcal{G}$ must be inverted to obtain the optimal stock demand, which is then decreasing over time. If $\pi^{*}(T)$ were larger than $\pi^{\infty}$, then the right branch of $\mathcal{G}$ would be relevant and the stock demand would be increasing. Notice that $\mathcal{G}$ is increasing before the pole $\pi^{\infty}$ and decreasing afterwards. This is the reason why the optimal stock demand is decreasing or increasing depending on whether $\pi^{\infty}>\pi^{*}(T)$ or $\pi^{\infty}<\pi^{*}(T)$.

For our benchmark calibration, Figure 2 depicts the optimal demand $m$ of an ordinary Merton problem and the optimal demands $\pi^{\infty}$ and $\pi^{*}(t)$ of the infinite-horizon as well as the finitehorizon problem. As expected for the finite-horizon problem, we obtain a stock demand that is decreasing over time. Since the initial time horizon is large, it starts close to the solution of the infinite-horizon case, which is about 0.57, and decreases at an increasing rate to the terminal condition $\pi^{*}(T)=0.3867$, i.e. the optimal stock demand is about $20 \%$ lower at the investment horizon. From an economic point of view, the time dependence comes from the fact that the relative price of liquidity, which is implicitly determined at the optimum, changes over time.

Figure 3 depicts the corresponding results if the stock demand in the Merton problem is $100 \%$. This is achieved by increasing the equity premium to 0.0867 , which is of course on high side. The case $m=1$ is particularly interesting since any derivation from $100 \%$ can be interpreted as resulting from the preferences for cash. The effect is about $7 \%$ for the infinite-horizon case, but is much more pronounced for the finite-horizon case. In fact, the optimal stock demand decreases from about $92 \%$ to $65 \%$ over time leading to a liquidity effect between $7 \%$ and $35 \%$. Figure 4 depicts a calibration where the Merton demand is $110 \%$. It can be seen that already in the infinite-horizon case preferences for cash bring down the optimal demand to less than 100\%. This is in line with Proposition 4.2. Otherwise, the demand would not be admissible. For the finite-horizon problem, the optimal stock demand decreases from $95 \%$ to $70 \%$.

Figure 5 shows comparative statics if we increase the weight of the cash preferences by decreasing the weight of consumption. Of course, the general effect is negative, i.e. more weight on 
cash preferences leads to less stock demand. However, it can be seen that the effect is initially pretty small, but at the end the terminal conditions are more sensitive. In particular, the optimal stock demands at the investment horizon vary between $38.7 \%$ and $6.8 \%$. Finally, Figure 6 depicts comparative statics if we vary the risk aversion coefficient. Now, all stock demands decrease in risk aversion, which is reasonable.

\section{Conclusion}

This paper studies a canonical consumption-portfolio problem with "money in the utility function". We provide the solution to the finite-horizon setting and analyze the relation to the problem with an infinite horizon. We show that for a finite horizon the stock demand can be increasing or decreasing over the life cycle where the latter result typically arises for reasonable calibrations. Therefore, adding preferences for cash to an ordinary Merton problem can generate life-cycle stock demands that are in line with rules of thumb for stock investing and recent empirical evidence. This could potentially be a very useful result for modeling life-cycle consumption-portfolio decisions.

\section{References}

Balvers, R. J., and D. Huang, 2009, Money and the C-CAPM, Journal of Financial and Quantitative Analysis 44, 337-368.

Brock, W. A., 1974, Money and growth: The case of long run perfect foresight, International Economic Review 15, 750-777.

Brown, S., W. Goetzmann, and S. A. Ross, 1995, Survival, Journal of Finance 50, 853-873.

Dixit, A. K., and S. M. Goldman, 1970, Uncertainty and the demand for liquid assets, Journal of Economic Theory 2, 368-382.

Duffie, D., 2001, Dynamic asset pricing theory, 3rd edn. Princeton University Press.

Fagereng, A., C. Gottlieb, and L. Guiso, 2017, Asset market participation and portfolio choice over the life-cycle, Journal of Finance 72, 705-750.

Fama, E., and A. Farber, 1979, Money, bonds, and foreign exchange, American Economic Review 69, 639-49. 
Fama, Eugene F., and Kenneth R. French, 2002, The Equity Premium, Journal of Finance 57, 637-659.

Gu, L., and D. Huang, 2013, Consumption, money, intratemporal substitution, and crosssectional asset returns, Journal of Financial Research 36, 115-146.

LeRoy, S. F., 1984a, Nominal prices and interest rates in general equilibrium: Endowment shocks, Journal of Business 57, 197-213.

LeRoy, S. F., 1984b, Nominal prices and interest rates in general equilibrium: Money shocks, Journal of Business 57, 177-195.

Lucas, R., 1982, Interest rates and currency prices in a two-country world, Journal of Monetary Economics 10, 335-359.

Merton, R. C., 1969, Lifetime portfolio selection under uncertainty: The continuous case, Review of Economics and Statistics 51, 247-257.

Merton, R. C., 1971, Optimal consumption and portfolio rules in a continuous-time model, Journal of Economic Theory 3, 373-413.

Munk, C., 2013, Financial asset pricing theory. Oxford University Press.

Obstfeld, M., and K. Rogoff, 1996, Foundations of international macroeconomics. MIT Press.

Sidrauski, Miguel, 1967, Rational choice and patterns of growth in a monetary economy, American Economic Review 57, 534-544.

Svensson, L., 1985, Money and asset prices in a cash-in-advance economy, Journal of Political Economy 93, 919-44. 


\section{A Proofs of Verification Results}

Proof of Theorem 6.3. It is sufficient to show that for all admissible controls $(\pi, c)$

$$
\int_{0}^{T} e^{-\delta s} \mathrm{E}\left[u\left(c_{s}, X_{s}^{\pi, c}\left(1-\pi_{s}\right)\right)\right] d s+e^{-\delta T} \mathrm{E}\left[U\left(X_{T}^{\pi, c}, X_{T}^{\pi, c}\left(1-\pi_{T}\right)\right)\right] \leq G\left(0, X_{0}\right)
$$

and for our candidate $\left(c^{*}, \pi^{*}\right)$

$$
\int_{0}^{T} e^{-\delta s} \mathrm{E}\left[u\left(c_{s}^{*}, X_{s}^{*}\left(1-\pi_{s}^{*}\right)\right)\right] d s+e^{-\delta T} \mathrm{E}\left[U\left(X_{T}^{*}, X_{T}^{*}\left(1-\pi_{T}^{*}\right)\right]=G\left(0, X_{0}\right)\right.
$$

where $X^{*} \equiv X^{\pi^{*}, c^{*}}$.

First, notice that, by Proposition 3.1, our candidates $\pi^{*}$ and $c^{*}$ are the global maximizers of the Bellman equation (3.5) for the relevant region $(\pi, c) \in(-\infty, 1) \times(0, \infty)$. Therefore, our candidates satisfy the Bellman equation (without supremum) as equality, whereas all other admissible controls satisfy the equation as inequality.

Second, we like to stress that a function $G$ satisfies the Bellman equation (3.5) if $V(t, x) \equiv$ $G(t, x) e^{-\delta t}$ satisfies

$$
0=\sup _{\pi, c}\left\{V_{t}+x r V_{x}+x \pi \eta V_{x}-c V_{x}+0.5 x^{2} \sigma^{2} \pi^{2} V_{x x}+e^{-\delta t} u(c, x(1-\pi))\right\}
$$

with terminal condition $V(T, x)=e^{-\delta T} G(T, x)$. Notice that the maximizers of the Bellman equation are not affected by this transformation.

For an admissible control, we now apply Ito's formula to $V\left(t, X_{t}\right)$

$$
\begin{array}{lll}
d V= & {\left[V_{t}+V_{x} X(r+\pi \eta-c / X)+0.5 V_{x x} X^{2} \sigma^{2} \pi^{2}\right] d t+V_{x} X \sigma \pi d W} \\
\stackrel{(A .46)}{=} & -e^{-\delta t} u(c, X(1-\pi)) d t+V_{x} X \sigma \pi d W
\end{array}
$$

where we omit all arguments. Evaluating at $t=T$ yields

$$
V\left(T, X_{T}\right)+\int_{0}^{T} e^{-\delta t} u\left(c_{t}, X_{t}\left(1-\pi_{t}\right)\right) d t \leq V\left(0, X_{0}\right)+\int_{0}^{T} V_{x}\left(t, X_{t}\right) X_{t} \sigma \pi_{t} d W_{t},
$$

where we have equality for $\left(\pi^{*}, c^{*}\right)$. The integrand of the stochastic integral

$$
V_{x}\left(t, X_{t}\right) X_{t} \sigma \pi_{t}=X_{t}^{1-\gamma} f^{k}(t) e^{-\delta t} \sigma \pi_{t}
$$

is an $\mathcal{L}^{2}$-process since $f$ is smooth and, by (iv) and (v) of an admissible strategy, $\pi$ and the coefficients of $X$ are bounded. Therefore, using the terminal condition and taking expectations yields

$$
\mathrm{E}\left[\int_{0}^{T} e^{-\delta t} u\left(c_{t}, X_{t}\left(1-\pi_{t}\right)\right) d t+e^{-\delta T} U\left(X_{T}, X_{T}\left(1-\pi_{T}\right)\right)\right] \leq V\left(0, X_{0}\right)
$$

with equality for $\left(\pi^{*}, c^{*}\right)$, which gives the desired result. 
Proof of Theorem 6.4. We must show that

$$
\int_{0}^{\infty} e^{-\delta t} \mathrm{E}\left[u\left(c_{t}, X_{t}^{\pi, c}\left(1-\pi_{t}\right)\right)\right] d t \leq G\left(X_{0}\right)
$$

for all admissible strategies and

$$
\int_{0}^{\infty} e^{-\delta t} \mathrm{E}\left[u\left(c^{\infty}, X_{t}^{*}\left(1-\pi^{\infty}\right)\right)\right] d t=G\left(X_{0}\right)
$$

for the optimal strategy where $X^{*} \equiv X^{\pi^{\infty}, c^{\infty}}$. As in the proof for the finite horizon, relation (A.47) still holds with $V(t, x)=G(x) e^{-\delta t}$. The integrand of the Ito integral is still an $\mathcal{L}^{2}$ process, i.e.

$$
\mathrm{E}\left[V\left(T, X_{T}^{\pi, c}\right)\right]+\mathrm{E}\left[\int_{0}^{T} e^{-\delta t} u\left(c_{t}, X_{t}^{\pi, c}\left(1-\pi_{t}\right)\right) d t\right] \leq V\left(0, X_{0}\right)
$$

or in terms of $G$

$$
e^{-\delta T} \mathrm{E}\left[G\left(X_{T}^{\pi, c}\right)\right]+\mathrm{E}\left[\int_{0}^{T} e^{-\delta t} u\left(c_{t}, X_{t}^{\pi, c}\left(1-\pi_{t}\right)\right) d t\right] \leq G\left(X_{0}\right) .
$$

Since the previous inequality holds for all admissible strategies $(\pi, c)$, we obtain

$$
\sup _{\pi, c}\left(e^{-\delta T} \mathrm{E}\left[G\left(X_{T}^{\pi, c}\right)\right]\right)+\mathrm{E}\left[\int_{0}^{T} e^{-\delta t} u\left(c_{t}, X_{t}^{\pi, c}\left(1-\pi_{t}\right)\right) d t\right] \leq G\left(X_{0}\right)
$$

If we multiply the term involving the integral by -1, the term becomes positive and we can apply the monotonous convergence theorem. Therefore,

$$
\lim _{T \rightarrow \infty} \mathrm{E}\left[\int_{0}^{T} e^{-\delta t} u\left(c_{t}, X_{t}\left(1-\pi_{t}\right)\right) d t\right]=\mathrm{E}\left[\int_{0}^{\infty} e^{-\delta t} u\left(c_{t}, X_{t}\left(1-\pi_{t}\right)\right) d t\right] .
$$

Hence, (A.48) follows if we can show that for every admissible strategy $(\pi, c)$

$$
\lim _{T \rightarrow \infty}\left(e^{-\delta T} \sup _{\pi, c} \mathrm{E}\left[G\left(X_{T}\right)\right]\right)=0
$$

holds. Set $\theta \equiv \eta / \sigma$. Then

$$
\begin{aligned}
0 & \geq \sup _{\pi, c}\left(e^{-\delta T} \mathrm{E}\left[G\left(X_{T}^{\pi, c}\right)\right]\right)=\sup _{\pi, c}\left(\mathrm{E}\left[\frac{1}{1-\gamma}\left(X_{T}^{\pi, c}\right)^{1-\gamma} f^{k}\right]\right) e^{-\delta T} \\
& \geq \underbrace{\sup _{\pi}\left(\mathrm{E}\left[\frac{1}{1-\gamma}\left(X_{T}^{\pi, 0}\right)^{1-\gamma}\right]\right)}_{(*)} e^{-\delta T} f^{k}=\underbrace{\frac{1}{1-\gamma}\left(X_{0}\right)^{1-\gamma} e^{(1-\gamma)\left(r+\frac{\theta^{2}}{2 \gamma}\right) T}}_{(*)} e^{-\delta T} f^{k} \longrightarrow 0 \text { for } T \rightarrow \infty,
\end{aligned}
$$

since (2.4) holds and $\gamma>1$. Notice that $(*)$ is the value function of an ordinary Merton problem with terminal wealth maximization only. One can check that it has the representation $(* *)$. 
Finally, we have to prove (A.49). Since (A.50) is satisfied as equality for the optimal strategy, it is sufficient to prove the transversality condition

$$
\lim _{T \rightarrow \infty}\left(e^{-\delta T} \mathrm{E}\left[G\left(X_{T}^{*}\right)\right]\right)=0,
$$

where

$$
d X^{*}=X^{*}\left[\left(r+\eta \pi^{\infty}-\omega^{\infty}\right) d t+\sigma \pi^{\infty} d W\right]
$$

and $\omega^{\infty} \equiv c^{\infty} / X^{*}$ is the consumption-wealth ratio which is given by (6.45). Therefore,

$$
e^{-\delta T} \mathrm{E}\left[G\left(X_{T}^{*}\right)\right]=\mathrm{E}\left[\frac{1}{1-\gamma}\left(X_{T}^{*}\right)^{1-\gamma} f^{k}\right] e^{-\delta T}=\frac{1}{1-\gamma} X_{0}^{1-\gamma} e^{\phi T} f^{k}
$$

where $\phi \equiv(1-\gamma)\left(r+\eta \pi^{\infty}-\omega^{\infty}-0.5 \gamma \sigma^{2}\left(\pi^{\infty}\right)^{2}\right)-\delta$ is a constant. It remains to show that

$$
\phi<0 .
$$

By (3.24), the optimal stock demand $\pi^{\infty}$ satisfies the equation

$$
\frac{P_{2}(\pi)}{\widetilde{K}}=-\frac{1-\beta}{\frac{1}{\gamma-1}+\beta}\left(r-\frac{\delta}{1-\gamma}+\left(\eta-0.5 \gamma \sigma^{2} \pi\right) \pi\right)+\left(\eta-\gamma \sigma^{2} \pi\right)(1-\pi)=0
$$

Therefore,

$$
\begin{aligned}
\phi & =(1-\gamma)\left(r-\frac{\delta}{1-\gamma}+\eta \pi^{\infty}-0.5 \gamma \sigma^{2}\left(\pi^{\infty}\right)^{2}-\frac{\beta}{1-\beta}\left(\eta-\gamma \sigma^{2} \pi^{\infty}\right)\left(1-\pi^{\infty}\right)\right) \\
& =\frac{(\gamma-1) \beta}{1-\beta}(-\frac{1-\beta}{\beta} \underbrace{\left[r-\frac{\delta}{1-\gamma}+\left(\eta-0.5 \gamma \sigma^{2} \pi^{\infty}\right) \pi^{\infty}\right]}_{(\times)}+\left(\eta-\gamma \sigma^{2} \pi^{\infty}\right)\left(1-\pi^{\infty}\right)) \\
& <\frac{(\gamma-1) \beta}{1-\beta} \frac{P_{2}\left(\pi^{\infty}\right)}{\widetilde{K}}=0,
\end{aligned}
$$

which shows (A.51). Notice that $(\times)$ is strictly positive since we assume (2.4) and $\pi^{\infty}>0$. 
Table 1: Constants. This table summarizes relevant constants that are defined in the paper.

\begin{tabular}{|c|c|c|c|}
\hline Name & & Definition & On Page \\
\hline$m$ & $\equiv$ & $\frac{\eta}{\gamma \sigma^{2}}$ & 3 \\
\hline$\kappa$ & $\equiv$ & $1+\beta(\gamma-1)$ & 4 \\
\hline$K$ & $\equiv$ & $\beta^{\frac{1}{\kappa}-1} \frac{\kappa}{1-\gamma}$ & 5 \\
\hline$\widetilde{K}$ & $\equiv$ & $K \frac{\beta}{1-\beta} \beta^{-\frac{1}{\kappa}}=\frac{\kappa}{(1-\gamma)(1-\beta)}$ & 7 \\
\hline$\kappa_{\alpha}$ & $\equiv$ & $1+\alpha(\gamma-1)$ & 8 \\
\hline$C$ & $\equiv$ & $\frac{1-\beta}{\beta} \beta^{\frac{1}{\kappa}}$ & 8 \\
\hline$a$ & $\equiv$ & $-(m+1)$ & 8 \\
\hline$b$ & $\equiv$ & $m-\frac{1-\beta}{\beta} \beta^{\frac{1}{\kappa}} \frac{1}{\gamma \sigma^{2}}$ & 8 \\
\hline$\widehat{K}$ & $\equiv$ & $\gamma \sigma^{2}(\widetilde{K}-0.5)$ & 9 \\
\hline$p$ & $\equiv$ & $\frac{\eta-\widetilde{K}\left(\eta+\gamma \sigma^{2}\right)}{\delta \widehat{K}}$ & 9 \\
\hline$q$ & $\equiv$ & $\frac{r-\frac{\delta}{1-\gamma}+\widetilde{K} \eta}{\widehat{K}}$ & 9 \\
\hline$D$ & $\equiv$ & $p^{2} / 4-q$ & 9 \\
\hline$\chi$ & $\equiv$ & $-\widetilde{K}=\frac{\kappa}{(\gamma-1)(1-\beta)}$ & 9 \\
\hline$\pi^{\infty}$ & $\equiv$ & $\pi_{02}=-0.5 p-\sqrt{D}$ & 10 \\
\hline $\bar{K}$ & $\equiv$ & $-\widehat{K} \frac{\gamma-1}{k}$ & 12 \\
\hline$\alpha_{1}$ & $\equiv$ & $\frac{1+\frac{\gamma}{k} m-\pi_{01}\left(1+\frac{\gamma}{k}\right)}{\left(\pi_{01}-\pi^{\infty}\right)\left(1-\pi_{01}\right)\left(\pi_{01}-m\right)}$ & 12 \\
\hline$\alpha_{2}$ & $\equiv$ & $\frac{1+\frac{\gamma}{k} m-\pi^{\infty}\left(1+\frac{\gamma}{k}\right)}{\left(\pi_{01}-\pi^{\infty}\right)\left(1-\pi^{\infty}\right)\left(m-\pi^{\infty}\right)}$ & 12 \\
\hline$\alpha_{3}$ & $\equiv$ & $\frac{\frac{\gamma}{k}}{\left(1-\pi_{01}\right)\left(1-\pi^{\infty}\right)}$ & 12 \\
\hline$\alpha_{4}$ & $\equiv$ & $\frac{1}{\left(\pi_{01}-m\right)\left(m-\pi^{\infty}\right)}$ & 12 \\
\hline
\end{tabular}


Table 2: Baseline parameter values. This table reports the baseline parameters.

\begin{tabular}{lcr}
\hline Symbol & Meaning & Value \\
\hline$r$ & Interest rate & 0.01 \\
$\eta$ & Equity premium & 0.05 \\
$\sigma$ & Stock volatility & 0.17 \\
$\gamma$ & Risk aversion & 3 \\
$\delta$ & Time preference rate & 0.03 \\
$\beta$ & Consumption weight & 0.99 \\
$\alpha$ & Consumption weight at death & 1.00 \\
\hline
\end{tabular}


Figure 1: The function $\mathcal{G}(\boldsymbol{\pi})$ in the baseline case. The figure depicts the function $\mathcal{G}(\pi)$. Baseline parameter values are used yielding a Merton result of $m=0.5767$. Besides, the terminal condition for the finite-horizon solution is $\pi^{*}(T)=0.3867$. The solution to the infinite-horizon problem $\pi^{\infty}=0.5696$ is the location of the pole of $\mathcal{G}$.

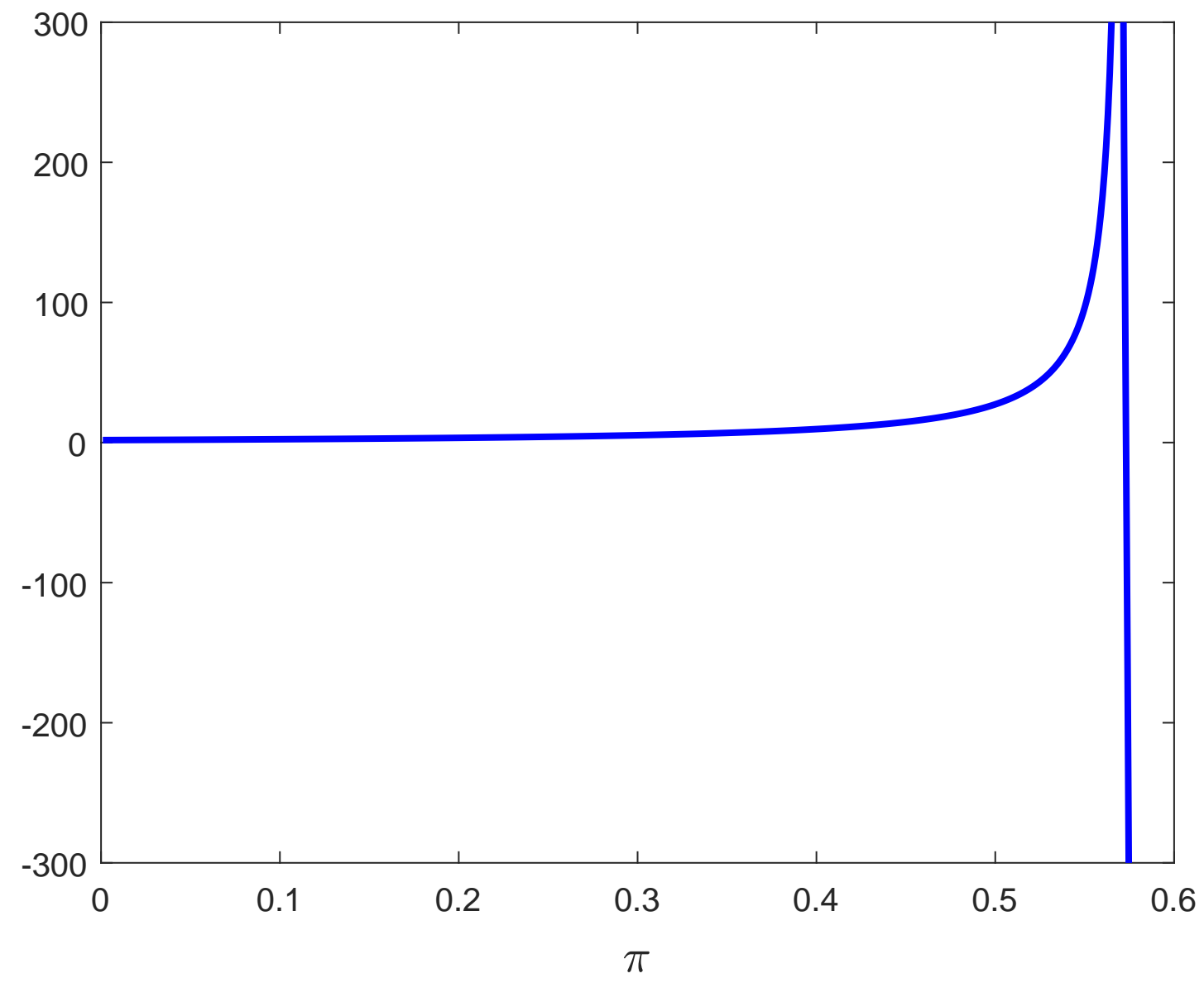


Figure 2: Optimal investments over the life cycle in the baseline case. The figure depicts the optimal proportion invested in stocks for the benchmark calibration reported in Table 2. It also shows the solution $m=0.5767$ to an ordinary Merton problem and the solution $\pi^{\infty}=0.5696$ to the problem with infinite horizon. The terminal condition for the finite-horizon solution is $\pi^{*}(T)=0.3867$.

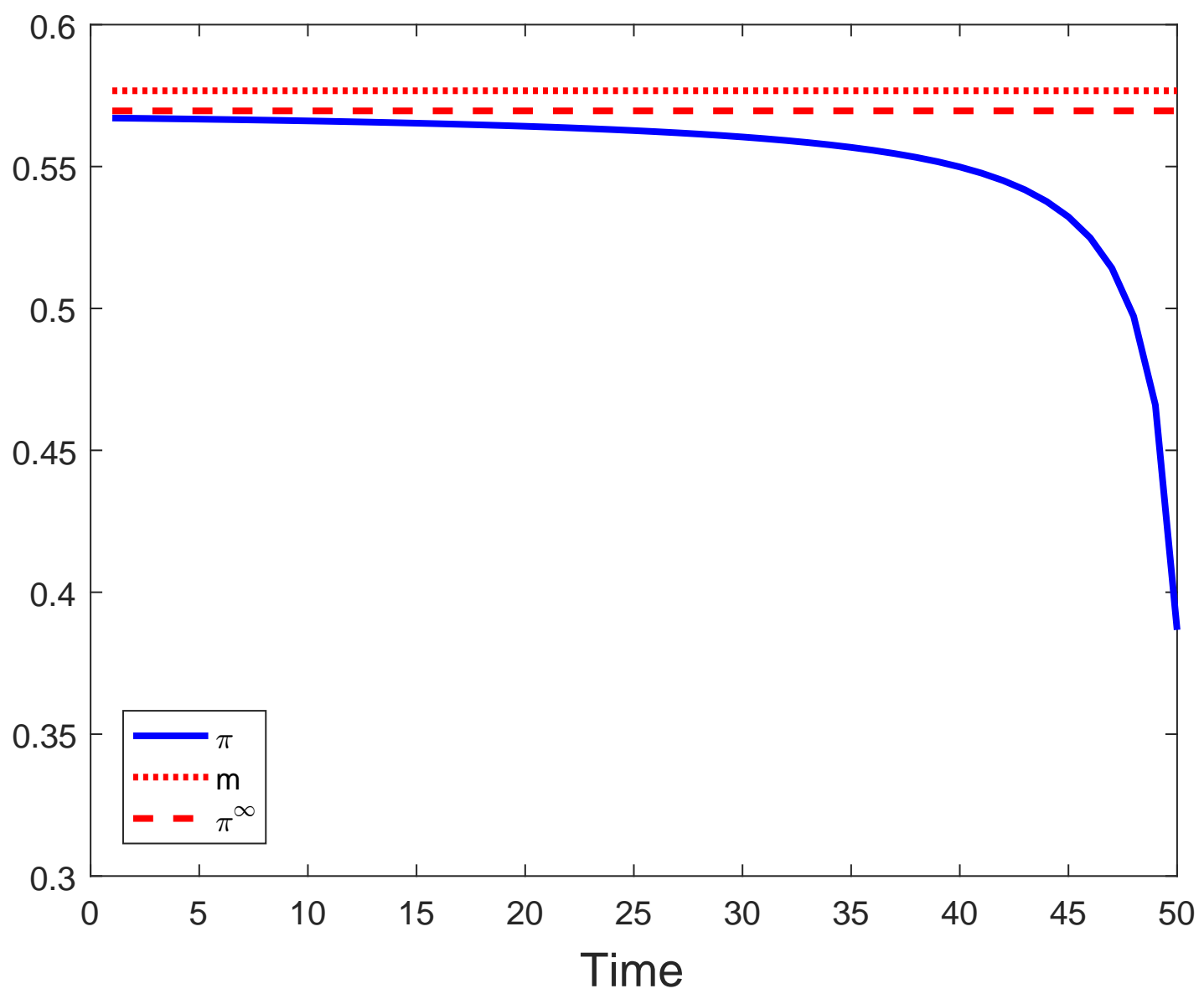


Figure 3: Optimal investments over the life cycle for $\boldsymbol{m}=1$. The figure depicts the optimal proportion invested in stocks for $m=1$. In this case, we increase the equity risk premium from $\eta=0.05$ in the benchmark case to $\eta=0.0867$. For the remaining parameters the baseline values are used. The solution to the infinite-horizon problem is $\pi^{\infty}=0.9274$. The terminal condition for the finite-horizon solution is $\pi^{*}(T)=0.6580$.

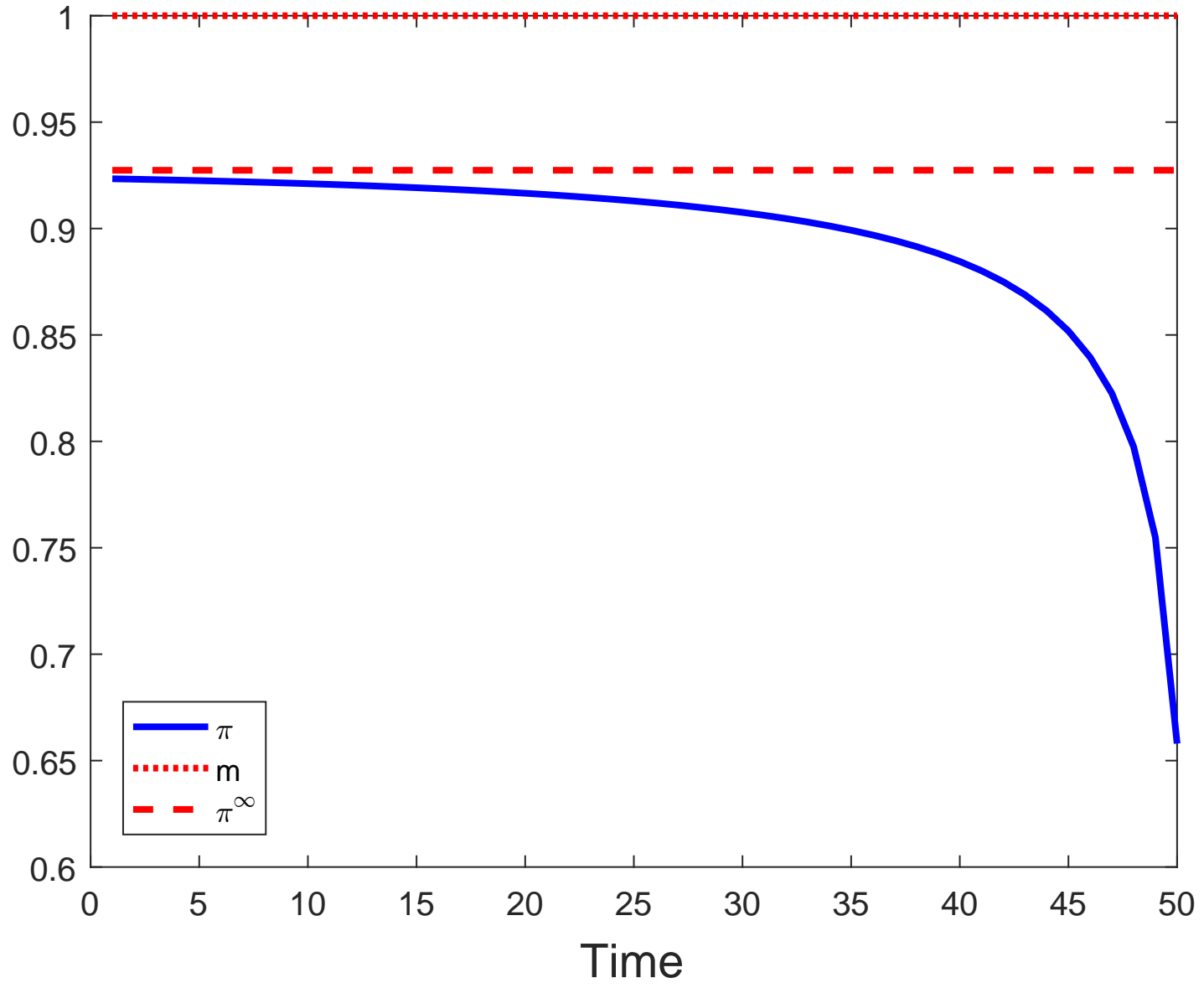


Figure 4: Optimal investments over the life cycle for $\boldsymbol{m}>\mathbf{1}$. The figure depicts the optimal proportion invested in stocks for $m>1$. In this case, we increase the equity risk premium from $\eta=0.05$ in the benchmark case to $\eta=0.09537$ so that $m=1.1$. For the remaining parameters the baseline values are used. The solution to the infinite-horizon problem is $\pi^{\infty}=0.9582$. The terminal condition for the finite-horizon solution is $\pi^{*}(T)=0.7042$.

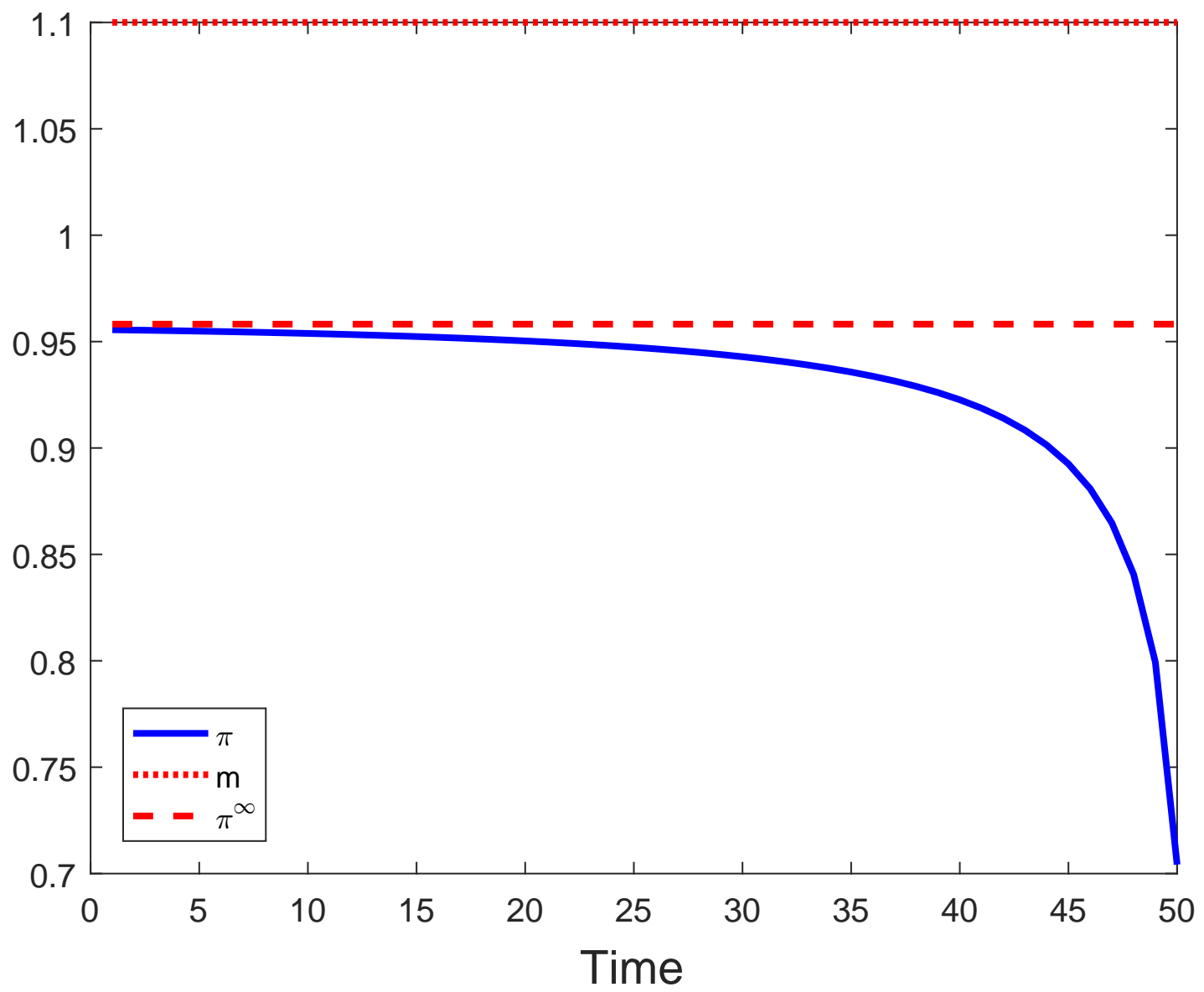


Figure 5: Optimal investments over the life cycle for different $\beta$. The figure depicts the optimal proportion invested in stocks for different values of $\beta$. For the remaining parameters the baseline values are used. The terminal conditions for the finite-horizon solutions are $\pi^{*}(T)=0.3867$ for $\beta=0.99, \pi^{*}(T)=0.2596$ for $\beta=0.98, \pi^{*}(T)=0.1566$ for $\beta=0.97$, and $\pi^{*}(T)=0.0675$ for $\beta=0.96$.
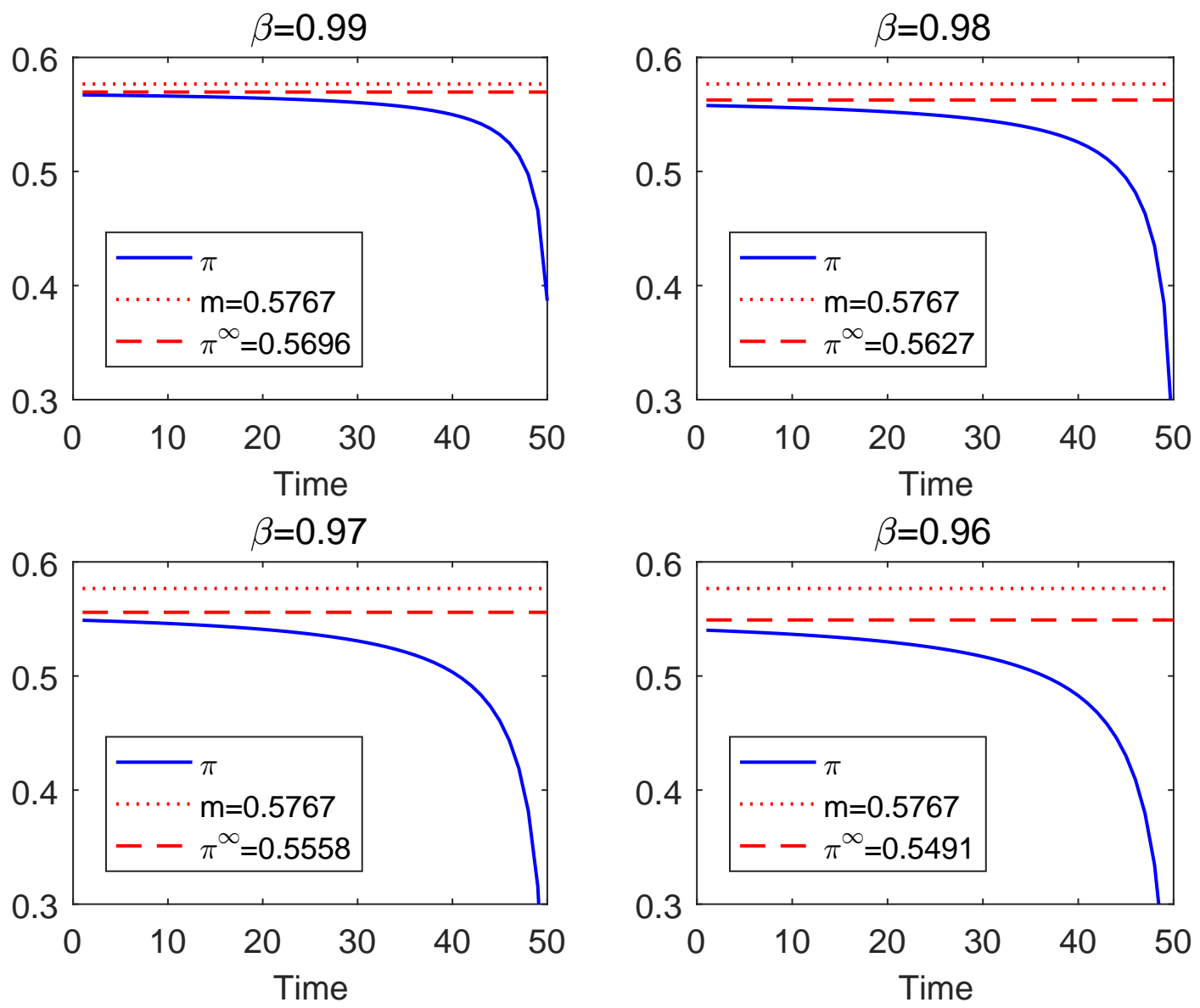
Figure 6: Optimal investments over the life cycle for different $\gamma$. The figure depicts the optimal proportion invested in stocks for different values of $\gamma$. For the remaining parameters the baseline values are used. The terminal conditions for the finite-horizon solutions are $\pi^{*}(T)=0.5888$ for $\gamma=1.5, \pi^{*}(T)=0.5094$ for $\gamma=2, \pi^{*}(T)=0.3867$ for $\gamma=3$, and $\pi^{*}(T)=0.3065$ for $\gamma=4$.
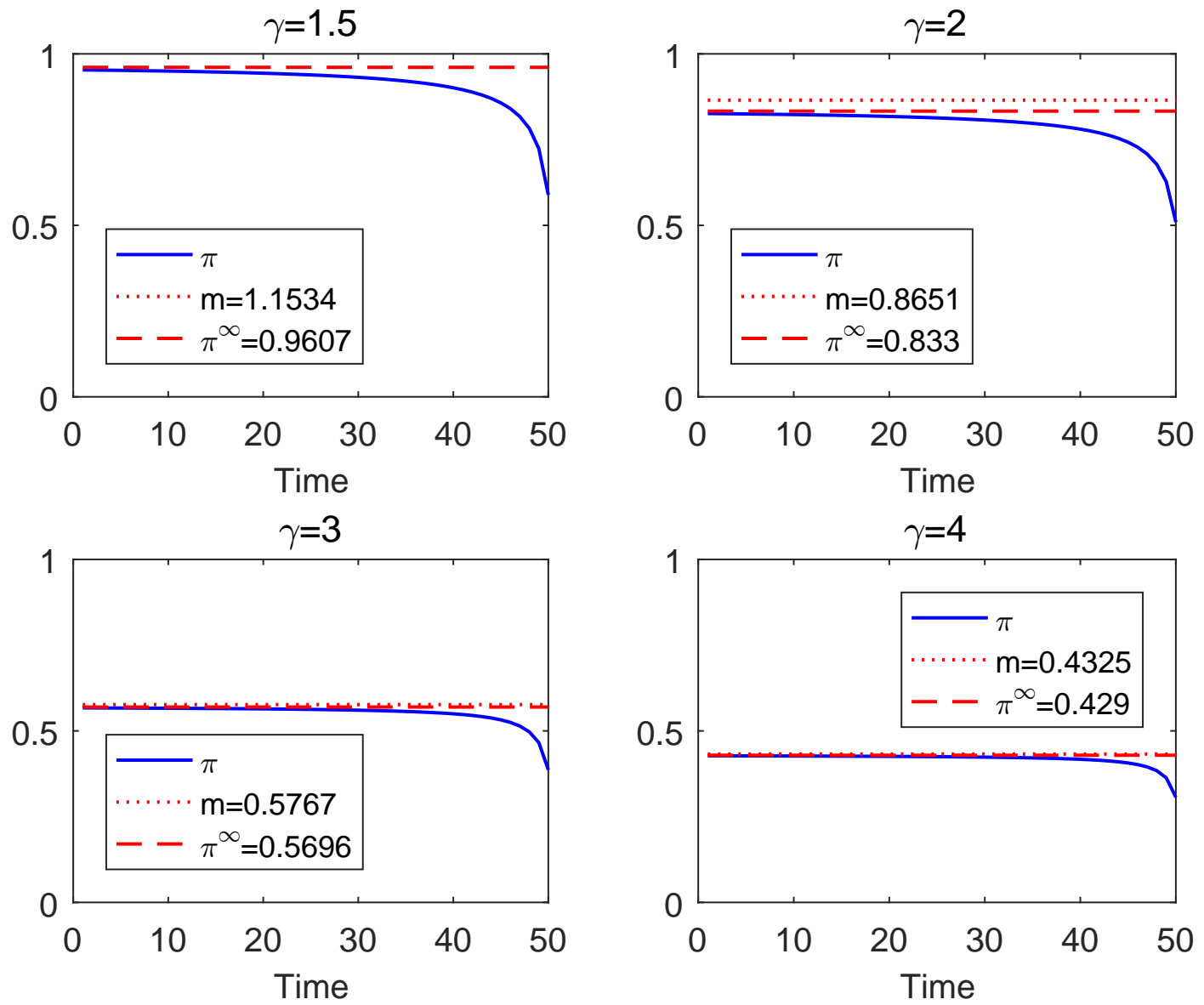


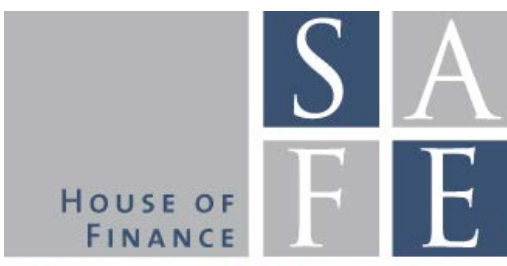

WORKING PAPER SERIES

\section{Recent Issues}

$\begin{array}{ll}\text { No. } 180 & \text { Tobias H. Tröger } \\ \text { No. } 179 & \text { Tobias H. Tröger }\end{array}$

No. 178 Matthias Goldmann

No. 177 Michael Donadelli, Marcus Jüppner, Max Riedel, Christian Schlag

No. 176 Giuliano Curatola, Ilya Dergunov

No. 175 Reint Gropp, Deyan Radev

No. 174 Reint Gropp, Deyan Radev

No. 173 Merlin Kuate Kamga, Christian Wilde

No. 172 Ahmed Khalifa, Massimiliano Caporin, Michele Costola, Shawkat Hammoudeh

No. 171 Michael Donadelli, Patrick Grüning

No. 169 Max Groneck, Alexander Ludwig, Alexander Zimper
Why MREL Won't Help Much

Too Complex to Work: A Critical Assessment of the Bail-in Tool under the European Bank Recovery and Resolution Regime

United in Diversity? The Relationship between Monetary Policy and Banking Supervision in the Banking Union

Temperature Shocks and Welfare Costs

International Capital Markets with TimeVarying Preferences

International Banking Conglomerates and the Transmission of Lending Shocks across Borders

Social Centralization, Bank Integration and the Transmission of Lending Shocks

Liquidity Premia in CDS Markets

Systemic Risk for Financial Institutions of Major Petroleum-based Economies: The Role of Oil

Innovation Dynamics and Fiscal Policy: Implications for Growth, Asset Prices, and Welfare

The Impact of Biases in Survival Beliefs on Savings Behavior 